\title{
Disease Severity at the First Hospitalization as a Predictor for Mechanical Ventilation Dependency in Elderly Patients with Chronic Obstructive Pulmonary Disease
}

\author{
Kuang-Ming Liao, ${ }^{1,2}$ Wei-Chieh Lin, ${ }^{3}$ Tzu-Chieh Lin, ${ }^{2}$ Chung-Yi Li ${ }^{4,5}$ and \\ Yea-Huei Kao Yang ${ }^{2}$ \\ ${ }^{1}$ Department of Internal Medicine, Chi Mei Medical Center, Tainan, Taiwan \\ ${ }^{2}$ Institute of Clinical Pharmacy and Pharmaceutical Sciences, National Cheng Kung University, Tainan, Taiwan \\ ${ }^{3}$ Medical Intensive Care Unit, Department of Internal Medicine, National Cheng Kung University Medical College \\ and Hospital, Tainan, Taiwan \\ ${ }^{4}$ Department of Public Health, College of Medicine, National Cheng Kung University, Tainan, Taiwan \\ ${ }^{5}$ Department of Public Health, College of Public Health, China Medical University, Taichung, Taiwan
}

\begin{abstract}
Patients with chronic obstructive pulmonary disease (COPD) are predisposed to respiratory failure with ventilator dependency. This study aims to determine the risk of prolonged mechanical ventilation (PMV), defined as 22 days or more of mechanical ventilation dependency after the first day of hospital admission (index date) in patients diagnosed with COPD. A retrospective cohort was conducted using medical claim data of Taiwan's National Health Insurance Research Database. Eligible study subjects were those who had a diagnosis of COPD made between January 1, 2005 and December 31, 2009. Patients were then followed until being registered as a PMV case, death, or the end of the study. The comorbidities were measured from January 1, 1997 to the index date by the ICD-9 code. The study sample consisted of 6,341 patients with COPD with a mean age of $73.89( \pm 12.01)$ years. Over a maximum of 6 years of follow-up, 654 patients developed PMV dependency, with an incidence density of 41.56 per 1,000 person-years. Patients aged 70 years and older were at significantly increased risk for PMV dependency, compared to those aged 40-49 years after adjusting for confounders. Expenses per visit and hospital visits per year, representing the severity of the COPD, were also associated with an elevated risk of PMV. Most patients developed PMV dependency within the first two years after the index date. Physicians should be aware that elderly patients with COPD have a high risk of PMV after first hospitalization, and these patients need to be closely monitored.
\end{abstract}

Keywords: chronic obstructive pulmonary disease; hospitalization; incidence; National Health Insurance Research Database; prolonged mechanical ventilation

Tohoku J. Exp. Med., 2014 December, 234 (4), 263-269. C C 2014 Tohoku University Medical Press

\section{Introduction}

Chronic obstructive pulmonary disease (COPD) is a progressive disease that is not fully reversible and is characterized by airflow limitation. It is also the major cause of mortality and morbidity in the world (Pauwels et al. 2001). As the disease progresses, patients may frequently suffer from acute exacerbation requiring hospitalization and possibly leading to respiratory failure and prolonged mechanical ventilation (PMV). PMV is defined as 22 days or more of mechanical ventilation dependency.

In addition, it has been shown that the number of patients requiring PMV has increased in Taiwan. There are approximately 30,000 patients who require PMV as recorded by the National Health Insurance (NHI) of Taiwan (Shih et al. 2013). The expenditure of these PMV-requiring patients is second only to cancer, accounting for approximately $20 \%$ of the total cost of catastrophic illness hospitalizations.

The growing burden of COPD raises the need for epidemiological COPD research to increase the understanding of the disease and its course (Vestbo et al. 2013), aid development of effective national health policies and facilitate equitable deployment of finite health-care resources in the prevention and management of COPD. We therefore aim to clarify the disease course of the patients requiring PMV following the first day of hospital admission due to COPD using the NHI database in Taiwan.

Received August 11, 2014; revised and accepted October 31, 2014. Published online November 28, 2014; doi: 10.1620/tjem.234.263.

Correspondence: Yea-Huei Kao Yang, Institute of Clinical Pharmacy and Pharmaceutical Sciences, National Cheng Kung University,

No.138, Shengli Rd., North Dist., Tainan City 704, Taiwan (R.O.C.).

e-mail: yhkao@mail.ncku.edu.tw 


\section{Materials and Methods}

Taiwan launched a single-payer NHI program in March 1995. More than $99 \%$ of Taiwanese citizens are enrolled in the program (Cheng et al. 2011). All of the medical institutions who are contracted with the NHI program must submit standard computerized claim documents for medical expenses. The NHI Research Database (NHIRD), a medical claims database, was established and used for research purposes. The NHIRD contains all of the claims data from the NHI Program in Taiwan, including patients' demographic characteristics, disease diagnoses, prescription records, and medical expenditures. In this study, we retrieved our study sample from 1 million beneficiaries randomly sampled from all of the beneficiaries registered in 2005. All of the medical claims from January 1, 1997 to December 31, 2010 for those 1 million beneficiaries are available. For data protection purposes, the confidential information of the individuals was scrambled and encrypted before the NHIRD released the information to the researchers; thus, informed consent forms were waived by Institutional Review Boards.

This study employed a retrospective cohort design in which eligible study subjects were patients who had a diagnosis of COPD (ICD-9-CM codes: 490-492, 496) and prescriptions for bronchodilating drugs, including $\beta 2$-agonists (short- and long-acting), anticholinergics, or theophylline during hospitalization between January 1, 2005 and December 31, 2009. Patients younger than 40 years and with a prior history of cancer were excluded. The patients hospitalized between January 1, 1997 and December 31, 2004 were further excluded to confirm the first hospitalization in the study period. All of the patients with COPD were then considered as the cases with a first admission for COPD during January 1, 2005 and December 31, 2009. The first date of COPD admission was defined as the index date. The patients were then followed from the index date to the end of 2010 to investigate the occurrence of onset of respiratory failure and PMV dependency ( $>21$ days). The comorbidities were measured from January 1, 1997 to the index date by the ICD-9 code in outpatient and inpatient department. Information on PMV-dependency was retrieved from the catastrophic illness document.

In the statistical analysis, we first described the demographic characteristics and co-morbidities of the study patients. The incidence density of PMV-dependence was estimated under Poisson assumption, and a life-table method (Cutler and Ederer 1958) was employed to estimate the cumulative incidence rate of PMV dependency over the study period. We used Cox's proportional hazard regression model with adjustment for potential confounders to assess the potential predictors for the onset of PMV dependency. The potential confounders included age, sex, geographic area, urbanization status, hospital visits per year, hospital length of stay per visit, expenses per visit and arrhythmia, coronary heart disease, hypertension, stroke, diabetes, depression and osteoporosis. Subjects who did not encounter PMV dependency before the end of study period (i.e., December $31,2010)$ were considered censored. The date of censoring was set on the last day of follow-up, which was considered either withdrawal from the NHI program or December 31, 2010. We also performed the $\log (-\log )$ test and confirmed that proportionality between hazards is assumed for the Cox model. All statistical analyses were performed using the Statistical Analysis Software (SAS) System, version 9.3 (SAS Institute Inc., Cary, NC, USA).

\section{Results}

There were 18,042 patients with COPD with hospitalization records from 1997 to 2009. Among them, we excluded the records of patients younger than 40 years $(n=$ $640)$, with malignant disease $(n=1,060)$, with COPD hospitalization coding from 1997 to $2004(n=9,801)$, and with a respiratory failure catastrophic illness document before December 31, $2004(n=200)$. The cohort comprised 6,341 patients. The mean age and corresponding standard deviation (S.D.) of the study population was $73.89( \pm 12.01)$ years and male dominated $(68.05 \%)$. More than $69 \%$ of patients were older than 70 years. Table 1 presents the distributions of demographic characteristics and selected comorbid medical disorders among the 6,341 COPD cases.

In this cohort, patients had a prevalence rate of coronary heart disease and depression of $58.02 \%$ and $84.01 \%$, respectively. The average number of hospital visits per year was 8.64 , and the average length of the hospital stay in the first hospital admission was 19.42 days. The average medical expenditure was 1,186 United States dollars per visit. The PMV events in this cohort population were 438 in men and 216 in women, and their incidence densities were 41.09 and 42.54 , respectively, per 1,000 patient-years. There was no significant difference between the gender groups with respect to PMV in patients with COPD after the first hospitalization. The most common reasons for patients' first hospitalization were chronic bronchitis and pneumonia; urinary tract infection also played an important role. Other causes of hospital admissions included heart failure, occlusion of cerebral arteries, and chronic ischemic heart disease (Table 2).

Table 3 showed the overall and specific incidence densities of respiratory failure in the study cohort. The incidence density rapidly increased in the group older than 70 years old with 56.8 per 1,000 patient-years. There was no significant difference in incidence density for gender, geographic area and urbanization status. During the follow-up period, most PMV cases occurred in the first 2 years $(77.52 \%)$ after the index date. The cumulative respiratory failure rate during 6 years was 18.55 per 100 person-years, and the cumulative survival rate was $81 \%$. A further subgroup analysis showed there was no significant difference in age below 70 years in cumulative respiratory failure. Subgroup analysis also showed most people who experienced PMV developed it in the first two years after the index date (Table 4).

Table 5 shows the sex, age, and urbanization statusspecific adjusted HRs for PMV. Potential confounders were adjusted for age, sex, geographic area, urbanization status, hospital visit per year, and length of hospital stay in the first hospital admission, expenses per visit, arrhythmia, coronary heart disease, hypertension, stroke, diabetes, depression and osteoporosis. During PMV, a significantly increased HR was observed for old age ( $\geq 70$ years old) (hazard ratio (HR) $=3.12,95 \%$ confidence interval $(\mathrm{CI})=1.90-5.14)$ and 
Table 1. Characteristics of the COPD patients' first hospitalizations in 2005-2009.

\begin{tabular}{|c|c|c|}
\hline & \multicolumn{2}{|c|}{ Study cohort } \\
\hline & $n$ & $\%$ \\
\hline \multicolumn{3}{|l|}{ Sex } \\
\hline Male & 4,315 & 68.05 \\
\hline Female & 2,026 & 31.95 \\
\hline \multicolumn{3}{|l|}{ Age (years) } \\
\hline $40-49$ & 316 & 4.98 \\
\hline $50-59$ & 622 & 9.81 \\
\hline $60-69$ & 1,027 & 16.20 \\
\hline $70-79$ & 2,172 & 34.25 \\
\hline$\geq 80$ & 2,204 & 34.76 \\
\hline Mean ( \pm S.D. $)$ & $73.89(12.01)$ & \\
\hline \multicolumn{3}{|l|}{ Urbanization status } \\
\hline Urban area & 2,015 & 31.78 \\
\hline Suburban area & 1,709 & 26.95 \\
\hline Rural area & 2,608 & 41.13 \\
\hline Missing & 9 & \\
\hline Hospital visit per year & $8.64(28.41)$ & \\
\hline \multicolumn{3}{|l|}{ Mean ( \pm S.D. $)$} \\
\hline Length of hospital stay in first hospital admission (days) Mean ( \pm S.D.) & $19.42(154.42)$ & \\
\hline Expenses per visit Mean ( \pm S.D.) (United States dollar) & $1,186(1,408)$ & \\
\hline \multicolumn{3}{|l|}{ Comorbidity } \\
\hline \multicolumn{3}{|l|}{ Arrhythmia } \\
\hline Yes & 2,281 & 35.97 \\
\hline No & 4,060 & 64.03 \\
\hline \multicolumn{3}{|l|}{ Coronary heart disease } \\
\hline Yes & 3,679 & 58.02 \\
\hline No & 2,662 & 41.98 \\
\hline \multicolumn{3}{|l|}{ Hypertension } \\
\hline Yes & 1,352 & 21.32 \\
\hline No & 4,989 & 78.68 \\
\hline \multicolumn{3}{|l|}{ Stroke } \\
\hline Yes & 2,119 & 33.42 \\
\hline No & 4,222 & 66.58 \\
\hline \multicolumn{3}{|l|}{ Diabetes } \\
\hline Yes & 2,330 & 36.74 \\
\hline No & 4,011 & 63.26 \\
\hline \multicolumn{3}{|l|}{ Depression } \\
\hline Yes & 5,327 & 84.01 \\
\hline No & 1,014 & 15.99 \\
\hline \multicolumn{3}{|l|}{ Osteoporosis } \\
\hline Yes & 1,567 & 24.71 \\
\hline No & 4,774 & 75.29 \\
\hline Total & 6,341 & 100.00 \\
\hline
\end{tabular}

slightly increased for hospital visit per year and expenses per visit.

\section{Discussion}

To our knowledge, this is the first study to analyze a nationwide PMV after first hospitalization in patients with
COPD to estimate the cumulative incidence rate stratified by age and observed years. This study shows that age more than 70 years at the time of the patient's first hospitalization, hospital visits per year, and expenses per visit to be the independent risk factors for PMV following the first hospital admission due to a variety of diseases. The incidence 
K.M. Liao et al.

Table 2. Cause of hospital admissions for patients with COPD first hospitalization.

\begin{tabular}{lccc}
\hline \multicolumn{1}{c}{ Disease } & Male & Female & Total $(\%=n / 6,341)$ \\
\hline Chronic bronchitis & 883 & 444 & $1,327(20.93)$ \\
Pneumonia & 611 & 200 & $811(12.79)$ \\
Chronic airways obstruction & 231 & 122 & $353(5.57)$ \\
Urinary tract infection & 127 & 153 & $280(4.42)$ \\
Other diseases of lung & 166 & 77 & $243(3.83)$ \\
Heart failure & 128 & 91 & $219(3.45)$ \\
Occlusion of cerebral arteries & 139 & 44 & $183(2.89)$ \\
Chronic ischemic heart disease & 109 & 44 & $153(2.41)$ \\
Septicemia & 86 & 53 & $139(2.19)$ \\
\hline
\end{tabular}

Table 3. Overall and specific incidence densities of respiratory failure with prolonged mechanical ventilation in the study cohort.

\begin{tabular}{lccc}
\hline & & \multicolumn{2}{c}{ COPD } \\
\cline { 2 - 4 } & No. of subjects & No. of events & ID $^{\mathrm{b}}\left(95 \% \mathrm{CI}^{\mathrm{c}}\right)$ \\
\hline Sex & & & \\
Male & 4,315 & 438 & $41.09(37.31-45.10)$ \\
Female & 2,026 & 216 & $42.54(37.05-8.60)$ \\
Age (years) & & & \\
$40-49$ & 316 & 16 & $15.32(8.76-24.88)$ \\
$50-59$ & 622 & 25 & $12.61(8.16-18.61)$ \\
$60-69$ & 1,027 & 66 & $21.44(16.58-27.28)$ \\
$70-79$ & 2,172 & 222 & $39.88(34.80-45.48)$ \\
$\geq 80$ & 2,204 & 325 & $79.98(71.52-89.16)$ \\
Urbanization status & & & $42.82(37.25-48.99)$ \\
Urban area & 2,015 & 212 & $40.33(34.51-46.84)$ \\
Suburban area & 1,709 & 171 & $41.53(36.70-46.75)$ \\
Rural area & 2,608 & 271 & $41.56(38.42-44.85)$ \\
Total & 6,341 & 654 & \\
\hline
\end{tabular}

anconsistency between the total population and the population summed for the individual variable was due to missing information.

${ }^{b} \mathrm{ID}$, incidence density (per 1,000 patient-years); CI, confidence interval.

'Based on poisson assumption.

rate of PMV increased in patients with COPD with age more than 70 years after adjusting for confounders. The cumulative incidence rate of PMV increased primarily within the first two years.

A previous study for the general population in Taiwan reported that the prevalence rate of PMV patients was 94.3 per 100,000 persons in 2004 , a rate that gradually declined to 89.38 per 100,000 persons by 2007 . The estimated mean \pm S.D. age was $77.99 \pm 13.87$ years, and $37.37 \%$ were women (Hung et al. 2013). In our study, the cumulative incidence of PMV in the first 2 years after the first hospitalization was 9.87 per 100 person-year in the COPD population, the mean age was 73.89 , and $31.95 \%$ were female. Our results indicate that patients with COPD who develop PMV are younger and more likely to be men than patients who develop PMV in the general population.
A previous report has also shown that the prevalence of PMV decreased in older patients. The number of PMV patients aged 75 years or older decreased from $74 \%$ in 2004 to $68 \%$ in 2007 , but this rate increased with age in the COPD population (Hung et al. 2013). Similarly, our study revealed that the cumulative incidence rate of PMV increased each year, especially for patients older than 70 years, compared to those aged $40-50$ years.

More than one-third of our population had other comorbidities, including coronary artery disease, stroke and diabetes, which may also contribute to PMV. The reported prevalence of diabetes among patients with COPD ranges from 1.6 to $16 \%$ in western countries, and there is evidence of an interaction between diabetes and COPD (Chatila et al. 2008). However, our study, which included patients with COPD with a first hospitalization, appeared to have higher 
Table 4. Follow-up period-specific cumulative incidence rate of respiratory failure with prolonged mechanical ventilation in the study cohort.

\begin{tabular}{|c|c|c|c|c|c|}
\hline $\begin{array}{l}\text { Period } \\
\text { (years) }\end{array}$ & No. of events & $\begin{array}{l}\text { Rate of respiratory } \\
\text { failure }\end{array}$ & Rate of survival & Cumulative survival & $\begin{array}{l}\text { Cumulative respiratory } \\
\text { failure (/100 person-year) }\end{array}$ \\
\hline $0-2$ & 507 & 0.10 & 0.90 & 0.90 & 9.87 \\
\hline $2-4$ & 103 & 0.04 & 0.96 & 0.86 & 13.57 \\
\hline $4-6$ & 44 & 0.06 & 0.94 & 0.81 & 18.55 \\
\hline \multicolumn{6}{|l|}{ Age $40-49$} \\
\hline $0-2$ & 14 & 0.05 & 0.95 & 0.95 & 4.96 \\
\hline $2-4$ & 2 & 0.01 & 0.99 & 0.94 & 6.02 \\
\hline $4-6$ & 0 & 0.00 & 1.00 & 0.94 & 6.02 \\
\hline \multicolumn{6}{|l|}{ Age 50-59 } \\
\hline $0-2$ & 20 & 0.04 & 0.96 & 0.96 & 3.70 \\
\hline $2-4$ & 2 & 0.01 & 0.99 & 0.96 & 4.28 \\
\hline $4-6$ & 3 & 0.03 & 0.97 & 0.93 & 6.85 \\
\hline \multicolumn{6}{|l|}{ Age $60-69$} \\
\hline $0-2$ & 50 & 0.06 & 0.94 & 0.94 & 5.73 \\
\hline $2-4$ & 9 & 0.02 & 0.98 & 0.93 & 7.40 \\
\hline $4-6$ & 7 & 0.04 & 0.96 & 0.89 & 11.08 \\
\hline \multicolumn{6}{|l|}{ Age $70-79$} \\
\hline $0-2$ & 169 & 0.09 & 0.91 & 0.91 & 9.45 \\
\hline $2-4$ & 37 & 0.04 & 0.96 & 0.87 & 13.19 \\
\hline $4-6$ & 16 & 0.06 & 0.94 & 0.82 & 18.37 \\
\hline \multicolumn{6}{|l|}{ Age $\geq 80$} \\
\hline $0-2$ & 254 & 0.15 & 0.85 & 0.85 & 15.35 \\
\hline $2-4$ & 53 & 0.09 & 0.91 & 0.77 & 22.96 \\
\hline $4-6$ & 18 & 0.12 & 0.88 & 0.68 & 32.49 \\
\hline
\end{tabular}

Table 5. Hazard ratios of respiratory failure with prolonged mechanical ventilation in relation to baseline characteristics of the study subjects, $2000-2010$.

\begin{tabular}{|c|c|c|c|}
\hline & Crude $\operatorname{HR}^{\mathrm{a}}(95 \% \mathrm{CI})$ & $\operatorname{AHR}^{\mathrm{b}}(95 \% \mathrm{CI})$ & $\operatorname{AHR}^{\mathrm{c}}(95 \% \mathrm{CI})$ \\
\hline \multicolumn{4}{|l|}{ Sex } \\
\hline Male & $0.96(0.81-1.13)$ & $1.08(0.91-1.27)$ & $1.07(0.89-1.27)$ \\
\hline \multicolumn{4}{|l|}{ Female (ref.) } \\
\hline \multicolumn{4}{|l|}{ Age (years) } \\
\hline \multicolumn{4}{|l|}{ 40-49 (ref.) } \\
\hline $50-59$ & $0.82(0.44-1.53)$ & $0.83(0.44-1.55)$ & $0.77(0.41-1.44)$ \\
\hline $60-69$ & $1.35(0.78-2.33)$ & $1.30(0.76-2.26)$ & $1.17(0.67-2.04)$ \\
\hline $70-79$ & $2.41(1.45-4.01)$ & $2.32(1.39-3.85)$ & $2.02(1.19-3.42)$ \\
\hline$\geq 80$ & $4.40(2.66-7.27)$ & $4.15(2.50-6.84)$ & $3.40(1.99-5.70)$ \\
\hline \multicolumn{4}{|l|}{ Urbanization status } \\
\hline Urban area & $1.03(0.86-1.23)$ & $1.15(0.94-1.41)$ & $1.09(0.89-1.34)$ \\
\hline Suburban area & $0.97(0.80-1.17)$ & $1.02(0.82-1.27)$ & $1.00(0.80-1.23)$ \\
\hline \multicolumn{4}{|l|}{ Rural area (ref.) } \\
\hline Hospital visits per year & $1.018(1.016-1.020)$ & $1.017(1.015-1.019)$ & $1.016(1.014-1.018)$ \\
\hline Length of hospital stay in first hospital admission & $1.004(1.000-1.009)$ & $1.005(0.999-1.011)$ & $1.003(0.995-1.010)$ \\
\hline Expenses per visit & $1.005(1.004-1.006)$ & $1.005(1.004-1.007)$ & $1.005(1.004-1.007)$ \\
\hline
\end{tabular}

${ }^{\mathrm{a}} \mathrm{HR}$, hazard ratio; $\mathrm{CI}$, confidence interval.

${ }^{\mathrm{b}} \mathrm{AHR}$, adjusted hazard ratio; based on Cox proportional hazard regression model with adjustment for age, sex, geographic area, urbanization status, hospital visit per year, length of hospital stay in first hospital admission, expenses per visit.

'AHR, adjusted hazard ratio; based on Cox proportional hazard regression model with adjustment for age, sex, geographic area, urbanization status, hospital visits per year, length of hospital stay in first hospital admission, expenses per visit and arrhythmia, coronary heart disease, hypertension, stroke, diabetes, depression, osteoporosis. 
prevalence of diabetes than previous studies. The prevalence of COPD and coronary heart disease is high in industrialized countries because they share common causal factors such as smoking. Similar to our findings showing a high prevalence $(58 \%)$ of coronary heart disease in our cohort, previous large population-based studies have suggested that patients with chronic bronchitis have a greater risk of coronary disease independent of the major cardiovascular risk factors (Jousilahti et al. 1996). In another prospective population study (Hole et al. 1996), patients with COPD were also shown to have a high mortality from ischemic heart disease. Furthermore, in comparison with previous studies that have identified clinically significant depressive symptoms in $42 \%-57 \%$ of patients with COPD, we found a dramatically higher prevalence $(84 \%)$ of depression in our cohort. Most of those studies were performed on small samples without control groups, using established diagnostic criteria, only clinical assessments or selfreported data, or containing no formal data analysis with differences in the objective characteristics and severity of COPD (Stage et al. 2006; Steer et al. 2010). In contrast, our study cohort is larger than those of previous reports, the data were retrieved from the COPD population that included patients hospitalized due to a variety of diseases, and the diagnosis of depression was made by a physician. Therefore, the high prevalence of depression in our patients can be explained by the occurrence of multiple comorbidities and the necessity of hospitalization.

A previous study (Donaldson and Wedzicha 2006) reviewed the published retrospective and prospective studies in patients with COPD identified with readmission due to COPD with acute exacerbation as a predicting factor for in-hospital and post-discharge mortality. However, to date, there are no data outlining the risk factors for PMV after the first hospitalization in patients with COPD. The growing burden of COPD demonstrates the need for more aggressive research and analysis of the epidemiology of COPD in order to raise awareness of the disease and its course, ensure the development of effective national health policies and facilitate equitable deployment of finite health-care resources in the prevention and management of COPD. In this study, we showed that the risk of PMV rapidly increased in the first 2 years after the first day of hospitalization in the COPD population, especially for those older than 70 years. In addition, Hung et al. (2011) examined the median survival, life expectancies, and cumulative incidence rate of patients undergoing PMV who were stratified by different underlying diseases and showed that life expectancies generally decreased with older age. In particular, patients with a combination diagnosis of septicemia and shock usually survived $<4$ months. Taken together, end-oflife care should be considered an important issue in elderly patients with COPD hospitalized for infectious diseases. The majority of hospital admissions for patients with COPD were due to acute exacerbations. The most common cause of acute exacerbations was respiratory tract infections. Of note, our data show that infection is also a common reason for the first hospitalization in the COPD population, including pneumonia $(n=811,12.79 \%)$, urinary tract infection $(n$ $=280,4.42 \%)$ and septicemia $(n=139,2.19 \%)$. The diseases associated with COPD, such as chronic bronchitis and chronic airway obstruction, remain the main reasons for the first hospitalization in patients with COPD. Respiratory tract infections, such as chronic bronchitis and pneumonia, during the first admission might be the important potential predictors of PMV.

Using a Cox proportional hazard regression model with adjustment for confounding factors, we found that hospital visits per year independently predicts PMV. This finding may reflect the underlying disease severity of COPD and the comorbidities. In comparison with a previous study showing 4.51 general practitioner visits per year in the 55-64 age range of patients with COPD (Cheng et al. 2011), our patients had more hospital visits per year. Aside from the older age for our patients, the convenience of medical care and the habit of hospital visitation in Taiwan may also contribute to this phenomenon.

Our study has several limitations that are confined by the database itself. First, the database did not contain any information regarding the disease severity, such as pulmonary function tests and clinical data of the PMV patients. We were therefore unable to further stratify the data by disease severity. We believe that this nationwide study with the entire patient population retrieved from the NHIRD database is capable of providing sufficient power to look into predictors for respiratory failure. There has been a study applying the NHIRD database to validate the results using clinical data and revealed concordance between clinical observation and retrospective conclusions (Cheng et al. 2011). Second, in order to more easily reimburse, it is possible that some diagnoses are over-represented to fulfill all of the reimbursement regulations of the NHI. However, the NHI of Taiwan has offered a list of major categories of catastrophic illnesses that are exempt from partial co-payments, including PMV, and each has its specific diagnostic criteria to prevent any abuse (Hung et al. 2011). The ICD-9 codes used for COPD diagnosis have been extensively employed in other studies (Wang et al. 2012), and the internal findings also support the coding validity (Wang et al. 2013). Third, the study design is retrospective; therefore, prospective studies are needed to further validate the conclusions. Each patient had a different enrollment time but the same end of PMV. Our data should therefore be interpreted as the description of PMV episodes after the first day of hospitalization in a given period of time. We believe that due to the number of enrolled participants and the comprehensive enrollment of hospitalization in Taiwan, the database is of normal distribution and the results are of statistical significance.

In conclusion, the patients with COPD experienced PMV after the first day of hospitalization with an incidence density of 41.56 per 1,000 person-years and a cumulative 
incidence rate of PMV that was increased primarily within the first two years. The study shows that age greater than 70 years, hospital visits per year, and expenses per visit are the independent risk factors for PMV following the first day of hospital admission due to a variety of diseases.

\section{Conflict of Interest}

The authors declare no conflict of interest.

\section{References}

Chatila, W.M., Thomashow, B.M., Minai, O.A., Criner, G.J. \& Make, B.J. (2008) Comorbidities in chronic obstructive pulmonary disease. Proc. Am. Thorac. Soc., 5, 549-555.

Cheng, C.L., Kao, Y.H., Lin, S.J., Lee, C.H. \& Lai, M.L. (2011) Validation of the National Health Insurance Research Database with ischemic stroke cases in Taiwan. Pharmacoepidemiol. Drug Saf., 20, 236-242.

Cutler, S.J. \& Ederer, F. (1958) Maximum utilization of the life table method in analyzing survival. J. Chronic Dis., 8, 699-712.

Donaldson, G.C. \& Wedzicha, J.A. (2006) COPD exacerbations. 1: Epidemiology. Thorax, 61, 164-168.

Hole, D.J., Watt, G.C., Davey-Smith, G., Hart, C.L., Gillis, C.R. \& Hawthorne, V.M. (1996) Impaired lung function and mortality risk in men and women: findings from the Renfrew and Paisley prospective population study. BMJ, 313, 711-715.

Hung, T.C., Lai, Y.F., Tseng, C.W., Hong, Y.H. \& Shi, H.Y. (2013) Trend analysis of hospital resource utilization for prolonged mechanical ventilation patients in Taiwan: a population-based study. Respir. Care, 58, 669-675.

Hung, M.C., Lu, H.M., Chen, L., Hu, F.C., Chan, S.Y., Yan, Y.H., Fan, P.S., Lin, M.S., Chen, C.R., Kuo, L.C., Yu, C.J. \& Wang, J.D. (2011) Life expectancies and incidence rates of patients under prolonged mechanical ventilation: a population-based study during 1998 to 2007 in Taiwan. Crit. Care, 15, R107.

Jousilahti, P., Vartiainen, E., Tuomilehto, J. \& Puska, P. (1996) Symptoms of chronic bronchitis and the risk of coronary disease. Lancet, $\mathbf{3 4 8}$, 567-572.

Pauwels, R.A., Buist, A.S., Calverley, P.M., Jenkins, C.R. \& Hurd, S. (2001) Global strategy for the diagnosis, management, and prevention of chronic obstructive pulmonary disease. NHLBI/ WHO Global Initiative for Chronic Obstructive Lung Disease (GOLD) Workshop summary. Am. J. Respir. Crit. Care Med., 163, 1256-1276

Shih, C.Y., Hung, M.C., Lu, H.M., Chen, L., Huang, S.J. \& Wang, J.D. (2013) Incidence, life expectancy and prognostic factors in cancer patients under prolonged mechanical ventilation: a nationwide analysis of 5,138 cases during 1998-2007. Crit. Care, 17, R144.

Stage, K.B., Middelboe, T., Stage, T.B. \& Sørensen, C.H. (2006) Depression in COPD: management and quality of life considerations. Int. J. Chron. Obstruct. Pulmon. Dis., 1, 315-320.

Steer, J., Gibson, G.J. \& Bourke, S.C. (2010) Predicting outcomes following hospitalization for acute exacerbations of COPD. QJM, 103, 817-829.

Vestbo, J., Hurd, S.S., Agustí, A.G., Jones, P.W., Vogelmeier, C., Anzueto, A., Barnes, P.J., Fabbri, L.M., Martinez, F.J., Nishimura, M., Stockley, R.A., Sin, D.D. \& Rodriguez-Roisin, R. (2013) Global strategy for the diagnosis, management, and prevention of chronic obstructive pulmonary disease: GOLD executive summary. Am. J. Respir. Crit. Care Med., 187, 347-365.

Wang, M.T., Lo, Y.W., Tsai, C.L., Chang, L.C., Malone, D.C., Chu, C.L. \& Liou, J.T. (2013) Statin use and risk of COPD exacerbation requiring hospitalization. Am. J. Med., 126, 598-606.

Wang, M.T., Tsai, C.L., Lo, Y.W., Liou, J.T., Lee, W.J. \& Lai, I.C. (2012) Risk of stroke associated with inhaled ipratropium bromide in chronic obstructive pulmonary disease: a population-based nested case-control study. Int. J. Cardiol., 158, 279-284. 